\title{
Nonexistence of classical diamagnetism and nonequilibrium fluctuation theorems for charged particles on a curved surface
}

\author{
Punyabrata Pradhan and Udo Seifert \\ II. Institut für Theoretische Physik, Universität Stuttgart, Stuttgart 70550, Germany \\ PACS 75.20.-g - Diamagnetism, paramagnetism, and superparamagnetism \\ PACS 05.40.-a - Fluctuation phenomena, random processes, noise, and Brownian motion
}

\begin{abstract}
We show that the classical Langevin dynamics for a charged particle on a closed curved surface in a time-independent magnetic field leads to the canonical distribution in the long time limit. Thus the Bohr-van Leeuwen theorem holds even for a finite system without any boundary and the average magnetic moment is zero. This is contrary to the recent claim by Kumar and Kumar (EPL, 86 (2009) 17001), obtained from numerical analysis of Langevin dynamics, that a classical charged particle on the surface of a sphere in the presence of a magnetic field has a nonzero average diamagnetic moment. We extend our analysis to a many-particle system on a curved surface and show that the nonequilibrium fluctuation theorems also hold in this geometry.
\end{abstract}

Introduction - The Bohr-van Leeuwen (BvL) theorem states that the average magnetic moment of a classical system consisting of charged particles in the presence of an external time-independent magnetic field is zero in thermal equilibrium [1. The proof is in principle simple: Since the free energy, calculated from the canonical partition func'tion, is independent of the external magnetic field, the average magnetic moment, which is the derivative of the free energy with respect to the magnetic field, is therefore identically zero.

At first glance the statement of the BvL theorem may appear disconcerting and counter-intuitive as, in the pres, ence of an external magnetic field, the charged particles undergo orbital motion. Thus, one could expect the system to have an average nonzero magnetic moment. But according to the BvL theorem, this is not the case. To understand the null classical magnetic moment physically, it is often pointed out that the boundary of a system plays a subtle role 114. It is argued that the charged particles in the bulk undergo orbital motion which gives rise to a nonzero diamagnetic moment, but there is also a paramagnetic moment arising due to incomplete orbits of particles which bounce off the boundary in a cuspidal manner. This paramagnetic contribution exactly cancels the diamagnetic one so that the net magnetic moment is zero. Recently, based on this intuitive picture, Kumar and $\mathrm{Ku}-$ mar have considered a finite system in the presence of a magnetic field [2] where there is no boundary, such as a case of a particle moving on the surface of a sphere.
These authors have claimed, from a numerical analysis of Langevin dynamics, that there exists a nonzero classical diamagnetic moment. It has been argued that the nonzero magnetic moment arises due to the avoided cancellation of the diamagnetic moment in the bulk and the paramagnetic moment having no contribution due to the absence of any boundary.

However, in this paper, we demonstrate that the above argument is incorrect. To this end, we consider a particle moving on a closed surface without any boundary in the presence of a constant magnetic field. We show analytically that, in the long time limit, the system which is governed by the classical Langevin dynamics is indeed described by the equilibrium canonical distribution. The case of a particle moving on a sphere, considered by Kumar and Kumar earlier [2], is one special case of a class of systems we study here. Recently, Kaplan and Mahanti 5] have considered Langevin dynamics of a particle constrained to move along a circle, but since in this case the motion of the particle is unaffected by the magnetic field, their analysis does not necessarily disprove the claim of Kumar and Kumar in full generality. However, motion on a sphere does depend on the magnetic field and therefore it is a first nontrivial case which will be studied here. Our results show that the previously claimed role of a boundary for having a zero diamagnetic moment is a misleading one because the average magnetic moment, as argued here, must vanish even for a finite boundary-less system which is shown to have a canonical distribution in thermal equi- 
librium. In principle, one can understand the reason for vanishing of the magnetic moment as following. In thermal equilibrium, the probabilities for a particle to have velocity $\vec{v}$ and $-\vec{v}$, at any given position $\vec{r}$, are equal and therefore the average magnetic moment $(q / 2 c)\langle\vec{r} \times \vec{v}\rangle$ of a particle of charge $q$ is zero.

We also generalize our results to a Newtonian manyparticle system with charged particles moving on a curved surface of an arbitrary shape in the presence of a timeindependent magnetic field. We show that Liouville's theorem holds in the absence of any thermal noise and damping. By adding those, the thermal distribution becomes the usual canonical one. At the end, we demonstrate with the Jarzynski equality [6] and the Crooks theorem [7] that paradigmatic cases of the nonequilibrium fluctuation theorems (reviewed in 8 [10]) are valid even for a system with particles on a curved surface in the presence of an external time-dependent magnetic field and other nonconservative forces. The fluctuation theorems in the presence of a time-dependent magnetic field in Euclidean space have been studied in [11,12.

Newtonian dynamics of a single particle - We start in the simplest setting where we first consider deterministic motion of a single particle of charge $q$ and mass $m$ moving in the presence of a uniform time-independent external magnetic field $\vec{B}$ applied along the $z$-axis. Newton's equation of motion can be simply written as,

$$
m \dot{\vec{v}}=\frac{q}{c}(\vec{v} \times \vec{B})
$$

where $\vec{v}$ the velocity of the particle and $\vec{B}=B \hat{z}, \hat{z}$ being the unit vector along the $z$-axis. In the presence of the constraint that the particle moves on the surface of a sphere with radius $a$, it is convenient to switch to spherical coordinate $\{r, \theta, \phi\}$ where the respective unit vectors are denoted as $\hat{r}, \hat{\theta}, \hat{\phi}$ and the constraint is expressed as $r=$ constant $=a$. By equating force and acceleration terms along $\hat{\theta}$ and $\hat{\phi}$ directions, Eq. (11) can be rewritten as

$$
\begin{gathered}
m a\left(\ddot{\theta}-\dot{\phi}^{2} \sin \theta \cos \theta\right) \hat{\theta}=\left(\frac{q B}{c} a \dot{\phi} \sin \theta \cos \theta\right) \hat{\theta}, \\
m a(\ddot{\phi} \sin \theta+2 \dot{\theta} \dot{\phi} \cos \theta) \hat{\phi}=-\left(\frac{q B}{c} a \dot{\theta} \cos \theta\right) \hat{\phi} .
\end{gathered}
$$

We convert these two second-order differential equations to four first-order differential equations in the 4dimensional phase space $\left\{\theta, \phi, v_{\theta}, v_{\phi}\right\}$,

$$
\begin{aligned}
\dot{\theta}=\frac{v_{\theta}}{a} & \equiv \mathcal{V}_{\theta}, \\
\dot{\phi}=\frac{v_{\phi}}{a \sin \theta} & \equiv \mathcal{V}_{\phi}, \\
\dot{v}_{\theta}=a\left[\frac{\cos \theta}{a^{2} \sin \theta} v_{\phi}^{2}+\frac{\omega_{c} \cos \theta}{a} v_{\phi}\right] & \equiv \mathcal{V}_{v_{\theta}}, \\
\dot{v}_{\phi}=-a\left[\frac{\cos \theta}{a^{2} \sin \theta} v_{\theta} v_{\phi}+\frac{\omega_{c} \cos \theta}{a} v_{\theta}\right] & \equiv \mathcal{V}_{v_{\phi}},
\end{aligned}
$$

using the relations $\ddot{\theta}=\dot{v}_{\theta} / a$ and $\ddot{\phi}=\left(\dot{v}_{\phi}-\right.$ $a \dot{\theta} \dot{\phi} \cos \theta) /(a \sin \theta)$ respectively in Eqs. (2) and (33), and writing the cyclotron frequency $w_{c}=q B / m c$.

The time evolution of the phase space density $\rho$, at a phase space point $\left\{\theta, \phi, v_{\theta}, v_{\phi}\right\}$, is governed by Liouville's equation which can be obtained from the equation of continuity in the phase space,

$$
\frac{\partial \rho}{\partial t}+\frac{\partial\left(\rho \mathcal{V}_{\theta}\right)}{\partial \theta}+\frac{\partial\left(\rho \mathcal{V}_{\phi}\right)}{\partial \phi}+\frac{\partial\left(\rho \mathcal{V}_{v_{\theta}}\right)}{\partial v_{\theta}}+\frac{\partial\left(\rho \mathcal{V}_{v_{\phi}}\right)}{\partial v_{\phi}}=0
$$

The time-independent solution of Liouville's equation, $\partial \rho_{\text {st }} / \partial t=0$, will be required later when we consider stochastic Langevin dynamics and deterministic manyparticle Newtonian dynamics of a system in the presence of a magnetic field. Since $\partial \mathcal{V}_{\theta} / \partial \theta=\partial \mathcal{V}_{\phi} / \partial \phi=$ $\partial \mathcal{V}_{v_{\theta}} / \partial v_{\theta}=0$ and $\partial \mathcal{V}_{v_{\phi}} / \partial v_{\phi}=-(\cos \theta / a \sin \theta) v_{\theta}$, the time-independent solution $\rho_{\text {st }}$ is simply given by

$$
\rho_{\text {st }}=\rho_{\text {mic }} \sin \theta=\frac{\sqrt{m}}{8 \pi^{2} a^{2} \sqrt{2 E}} \sin \theta,
$$

where the numerical value of the microcanonical probability distribution $\rho_{\text {mic }}$ follows from the normalization condition

$$
\int d \Lambda\left(\theta, \phi, v_{\theta}, v_{\phi}\right) \rho_{\mathrm{mic}} \delta\left(m\left(v_{\theta}^{2}+v_{\phi}^{2}\right) / 2-E\right)=1
$$

with $\int d \Lambda=a^{2} \int_{0}^{\pi} \sin \theta d \theta \int_{0}^{2 \pi} d \phi \int_{-\infty}^{\infty} d v_{\theta} \int_{-\infty}^{\infty} d v_{\phi}$ denoting integration over volume in the phase space $\left\{\theta, \phi, v_{\theta}, v_{\phi}\right\}$ and $E$ the total kinetic energy of the particle. Clearly the factor of $\sin \theta$ in Eq. (9) comes from the measure of an infinitesimal area on the surface of a sphere in spherical coordinate. The statement of Liouville's theorem that the phase space is incompressible under Newtonian time-evolution can now be expressed as $d \tilde{\rho} / d t=0$ where $\tilde{\rho}=(\rho / \sin \theta)$. Note that the timeindependent magnetic field does not perform any work (nor do the constraint-forces) on the particle, implying $\mathrm{d} E / \mathrm{d} t=m\left(v_{\theta} \dot{v}_{\theta}+v_{\phi} \dot{v}_{\phi}\right)=0$, and using Eqs. (6) and (7), we get

$$
\left(v_{\theta} \mathcal{V}_{v_{\theta}}+v_{\phi} \mathcal{V}_{v_{\phi}}\right)=0
$$

Langevin dynamics - Now we analyse the problem considered in Ref. 2], i.e., the classical Langevin dynamics of a charged particle constrained to move on the surface of a sphere in the presence of an external uniform magnetic field. The Langevin equations are obtained by adding a noise term and a friction force to Newton's equations, Eqs. (6) and (7),

$$
\begin{aligned}
\dot{v}_{\theta} & =\mathcal{V}_{v_{\theta}}-\gamma v_{\theta}+\sqrt{2 \gamma k_{B} T / m} f_{\theta}, \\
\dot{v}_{\phi} & =\mathcal{V}_{v_{\phi}}-\gamma v_{\phi}+\sqrt{2 \gamma k_{B} T / m} f_{\phi},
\end{aligned}
$$

in addition to Eqs. (4) and (5), where $\gamma$ is the friction coefficient, $k_{B}$ is the Boltzmann constant, $T$ is the temperature, $\vec{f}$ is the Gaussian white-noise satisfying the fluctuation-dissipation theorem, $\left\langle f_{\alpha}(t) f_{\alpha^{\prime}}\left(t^{\prime}\right)\right\rangle=\delta_{\alpha \alpha^{\prime}} \delta(t-$ 
$\left.t^{\prime}\right)$ and the subscript corresponds to the component along unit vector $\hat{\alpha}$ or $\hat{\alpha}^{\prime}$ with $\alpha, \alpha^{\prime} \equiv \theta, \phi$. The probability density $P\left(\theta, \phi, v_{\theta}, v_{\phi}\right)$ is governed by the Fokker-Planck equation [13],

$$
\begin{gathered}
\frac{\partial P}{\partial t}=-\frac{\partial\left(\mathcal{V}_{\theta} P\right)}{\partial \theta}-\frac{\partial\left(\mathcal{V}_{\phi} P\right)}{\partial \phi}-\frac{\partial\left(\mathcal{V}_{v_{\theta}} P\right)}{\partial v_{\theta}}-\frac{\partial\left(\mathcal{V}_{v_{\phi}} P\right)}{\partial v_{\phi}} \\
+\frac{\partial\left(\gamma v_{\theta} P\right)}{\partial v_{\theta}}+\frac{\partial\left(\gamma v_{\phi} P\right)}{\partial v_{\phi}}+\frac{\gamma k_{B} T}{m}\left[\frac{\partial^{2} P}{\partial v_{\theta}^{2}}+\frac{\partial^{2} P}{\partial v_{\phi}^{2}}\right] .
\end{gathered}
$$

To solve the steady state of the above equation, i.e. the solution for which $\partial P_{\mathrm{st}} / \partial t=0$, we try the equilibrium ansatz $P_{\mathrm{st}}\left(\theta, \phi, v_{\theta}, v_{\phi}\right)=$ const $\times \rho_{\mathrm{st}} \times P_{1}\left(v_{\theta}, v_{\phi}\right)$ where $\rho_{\mathrm{st}}$ is the time-independent solution of Eq. (8) and $P_{1}\left(v_{\theta}, v_{\phi}\right)=\exp \left[-m\left(v_{\theta}^{2}+v_{\phi}^{2}\right) / 2 k_{B} T\right]$. Using $\partial P_{1} / \partial v_{\theta}=$ $-\left(m / k_{B} T\right) v_{\theta} P_{1}, \partial P_{1} / \partial v_{\phi}=-\left(m / k_{B} T\right) v_{\phi} P_{1}$ and Eq. (11), the steady-state probability distribution for a particle moving on the surface of a sphere turns out to be the equilibrium canonical probability distribution function,

$$
\rho_{\text {can }}\left(\theta, \phi, v_{\theta}, v_{\phi}\right)=\frac{P_{\mathrm{st}}}{\sin \theta}=\frac{m}{8 \pi^{2} a^{2} k_{B} T} e^{-\mathcal{E} / k_{B} T},
$$

where $\mathcal{E}=m\left(v_{\theta}^{2}+v_{\phi}^{2}\right) / 2$ and the normalization condition being $\int d \Lambda \rho_{\text {can }}=1$. The probability of finding the particle at any point of the sphere is uniform. According to the general theory of Fokker-Planck equations [13, any initial distribution will relax to a unique stationary distribution, i.e., in this case the canonical one, in the long time limit. Thus one can see that the average magnetic moment is zero since $\left\langle v_{\theta}\right\rangle=\left\langle v_{\phi}\right\rangle=0$ with this distribution, and hence there is no classical diamagnetism.

Langevin dynamics with an external potential - The above analysis can be easily extended to the case when there is an external potential $U(\vec{r})$ at a position $\vec{r}$ due to conservative forces in addition to an external magnetic field. The Langevin equation is then written as

$$
m \dot{\vec{v}}=q(\vec{v} \times \vec{B}) / c-\vec{\nabla} U-\gamma m \vec{v}+\sqrt{2 \gamma m k_{B} T} \vec{f},
$$

where $\vec{f}$ is the Gaussian white-noise satisfying the fluctuation-dissipation theorem. When the particle is constrained to move on the surface of a sphere, Eq. (16) can be rewritten by equating respective components of forces and accelerations along $\hat{\theta}$ and $\hat{\phi}$ directions,

$$
\begin{aligned}
\dot{v}_{\theta} & =\mathcal{V}_{v_{\theta}}-(\vec{\nabla} U)_{\theta} / m-\gamma v_{\theta}+\sqrt{2 \gamma k_{B} T / m} f_{\theta}, \\
\dot{v}_{\phi} & =\mathcal{V}_{v_{\phi}}-(\vec{\nabla} U)_{\phi} / m-\gamma v_{\phi}+\sqrt{2 \gamma k_{B} T / m} f_{\phi},
\end{aligned}
$$

where the gradient $\vec{\nabla} U(\vec{r})$ is expressed in spherical coordinate with $(\vec{\nabla} U)_{\theta}=(1 / a)(\partial U / \partial \theta)$ and $(\vec{\nabla} U)_{\phi}=$ $(1 / a \sin \theta)(\partial U / \partial \phi)$ respectively being the $\hat{\theta}$ and $\hat{\phi}$ components of the external conservative-forces. The full set of Langevin equations now consists of Eqs. (4), (5), (17) and (18). The corresponding Fokker-Planck equation can be written as

$$
\frac{\partial P}{\partial t}=-\frac{\partial\left(\mathcal{V}_{\theta} P\right)}{\partial \theta}-\frac{\partial\left(\mathcal{V}_{\phi} P\right)}{\partial \phi}-\frac{\partial\left(\mathcal{V}_{v_{\theta}} P\right)}{\partial v_{\theta}}-\frac{\partial\left(\mathcal{V}_{v_{\phi}} P\right)}{\partial v_{\phi}}
$$

$$
\begin{array}{r}
+\frac{\partial\left(\frac{1}{m}(\vec{\nabla} U)_{\theta} P\right)}{\partial v_{\theta}}+\frac{\partial\left(\frac{1}{m}(\vec{\nabla} U)_{\phi} P\right)}{\partial v_{\phi}} \\
+\frac{\partial\left(\gamma v_{\theta} P\right)}{\partial v_{\theta}}+\frac{\partial\left(\gamma v_{\phi} P\right)}{\partial v_{\phi}}+\frac{\gamma k_{B} T}{m}\left[\frac{\partial^{2} P}{\partial v_{\theta}^{2}}+\frac{\partial^{2} P}{\partial v_{\phi}^{2}}\right] .
\end{array}
$$

It is straightforward to show that the time-independent solution of the Fokker-Planck equation, $\partial P_{\mathrm{st}} / \partial t=0$, can be written as $P_{\text {st }}=(\sin \theta) \rho_{\text {can }}$ where the canonical probability distribution $\rho_{\text {can }}$ is given by

$$
\rho_{\text {can }}\left(\theta, \phi, v_{\theta}, v_{\phi}\right)=\left(e^{-\mathcal{E} / k_{B} T}\right) / Z
$$

with the total energy $\mathcal{E}=\left[m\left(v_{\theta}^{2}+v_{\phi}^{2}\right) / 2+U(\theta, \phi)\right]$ and the partition function $Z=\int d \Lambda \exp \left(-\mathcal{E} / k_{B} T\right)$. Clearly, the probability distribution, and therefore the partition function, are both independent of the magnetic field.

Newtonian dynamics of a many-particle system - Now we consider a classical Newtonian system consisting of $N$ interacting charged particles, with mass $m$ and charge $q$, moving on the surface of a sphere in the presence of a constant external magnetic field $\vec{B}(\vec{r})$ which may be spacedependent. Newton's equation of motion of the $i$-th particle can be written as

$$
\left(m \dot{\vec{v}}_{i}\right)_{\hat{\alpha}}=\left(\frac{q}{c}\left(\vec{v}_{i} \times \vec{B}\left(\vec{r}_{i}\right)\right)\right)_{\hat{\alpha}}-\left(\vec{\nabla}_{\vec{r}_{i}} U\left(\left\{\vec{r}_{i}\right\}\right)\right)_{\hat{\alpha}}
$$

where $\vec{r}_{i}, \vec{v}_{i}$ position and velocity of the $i$-the particle, $(\ldots)_{\hat{\alpha}}$ denotes a component along the unit vector $\hat{\alpha}$ with $\alpha \equiv \theta, \phi$, and $U\left(\left\{\vec{r}_{i}\right\}\right)$ is the scalar potential containing both internal pair-potentials as well as an external potential. Similarly as in the previous single-particle case of Eqs. (4)-(77), Newton's equations of motion can be written in spherical coordinate in $4 N$-dimensional phase space $\left\{\theta_{i}, \phi_{i}, v_{\theta_{i}}, v_{\phi_{i}}\right\}$ as

$$
\begin{array}{r}
\dot{\theta}_{i}=\frac{v_{\theta_{i}}}{a} \equiv \mathcal{V}_{\theta}^{(i)}, \\
\dot{\phi}_{i}=\frac{v_{\phi_{i}}}{a \sin \theta_{i}} \equiv \mathcal{V}_{\phi}^{(i)}, \\
\dot{v}_{\theta_{i}}=\mathcal{V}_{v_{\theta}}^{(i)}-\frac{1}{m}\left(\vec{\nabla}_{\vec{r}_{i}} U\left(\left\{\vec{r}_{i}\right\}\right)\right)_{\hat{\theta}_{i}}, \\
\dot{v}_{\phi_{i}}=\mathcal{V}_{v_{\phi}}^{(i)}-\frac{1}{m}\left(\vec{\nabla}_{\vec{r}_{i}} U\left(\left\{\vec{r}_{i}\right\}\right)\right)_{\hat{\phi}_{i}},
\end{array}
$$

where $\mathcal{V}_{v_{\theta}}^{(i)}=a\left[\left(\cos \theta_{i} / a^{2} \sin \theta_{i}\right) v_{\phi_{i}}^{2}+\left(\omega_{c} \cos \theta_{i} / a\right) v_{\phi_{i}}\right]$ and $\left(\vec{\nabla}_{\vec{r}_{i}} U\left(\left\{\vec{r}_{i}\right\}\right)\right)_{\hat{\theta}_{i}}=(1 / a)\left(\partial U\left(\left\{\theta_{i}, \phi_{i}\right\}\right) / \partial \theta_{i}\right)$, $\mathcal{V}_{v_{\phi}}^{(i)}=-a\left[\left(\cos \theta_{i} / a^{2} \sin \theta_{i}\right) v_{\theta_{i}} v_{\phi_{i}}+\left(\omega_{c} \cos \theta_{i} / a\right) v_{\theta_{i}}\right]$ and $\left(\vec{\nabla}_{\vec{r}_{i}} U\left(\left\{\vec{r}_{i}\right\}\right)\right)_{\hat{\phi}_{i}}=\left(1 / a \sin \theta_{i}\right)\left(\partial U\left(\left\{\theta_{i}, \phi_{i}\right\}\right) / \partial \phi_{i}\right)$. The time evolution of the phase space density $\rho\left(\left\{\theta_{i}, \phi_{i}, v_{\theta_{i}}, v_{\phi_{i}}\right\}\right)$ in $4 N$-dimensional phase space is given by the continuity equation,

$\frac{\partial \rho}{\partial t}+\sum_{i=1}^{N}\left[\frac{\partial\left(\rho \mathcal{V}_{\theta}^{(i)}\right)}{\partial \theta_{i}}+\frac{\partial\left(\rho \mathcal{V}_{\phi}^{(i)}\right)}{\partial \phi_{i}}+\frac{\partial\left(\rho \mathcal{V}_{v_{\theta}}^{(i)}\right)}{\partial v_{\theta_{i}}}+\frac{\partial\left(\rho \mathcal{V}_{v_{\phi}}^{(i)}\right)}{\partial v_{\phi_{i}}}\right]$ $=0 .(26)$ 
Using $\partial \mathcal{V}_{\theta}^{(i)} / \partial \theta_{i}=\partial \mathcal{V}_{\phi}^{(i)} / \partial \phi_{i}=\partial \mathcal{V}_{v_{\theta}}^{(i)} / \partial v_{\theta_{i}}=0$ and $\partial \mathcal{V}_{v_{\phi}}^{(i)} / \partial v_{\phi_{i}}=-\left(\cos \theta_{i} / a \sin \theta_{i}\right) v_{\theta_{i}}$, it is straightforward to see that the time-independent solution of Eq. (26), $\partial \rho_{\text {st }} / \partial t=0$, is given by

$$
\rho_{\mathrm{st}}\left(\left\{\theta_{i}, \phi_{i}, v_{\theta_{i}}, v_{\phi_{i}}\right\}\right)=\rho_{\text {mic }}\left(\prod_{i=1}^{N} \sin \theta_{i}\right) .
$$

The microcanonical probability measure is $\rho_{\text {mic }}=1 / \Omega(E)=$ const and $\Omega(E)$ is the total phase volume of a constant energy surface in $4 N$-dimensional phase space i.e., $\Omega(E)=$ $\prod_{i}\left(\int d \Lambda\left(\theta_{i}, \phi_{i}, v_{\theta_{i}}, v_{\phi_{i}}\right)\right) \delta\left(\mathcal{E}\left(\left\{\theta_{i}, \phi_{i}, v_{\theta_{i}}, v_{\phi_{i}}\right\}\right) \quad\right.$ - E $\left.E\right)$ where $d \Lambda$ is the infinitesimal volume-measure as defined below Eq. (10) and the total energy of the system is $\mathcal{E}=\left[(m / 2) \sum_{i}\left(v_{\theta_{i}}^{2}+v_{\phi_{i}}^{2}\right)+U\left(\left\{\theta_{i}, \phi_{i}\right\}\right)\right]$. Also note that $d \tilde{\rho} / d t=0$ with $\tilde{\rho}=\rho /\left(\prod_{i} \sin \theta_{i}\right)$, which is the statement of Liouville's theorem. Therefore, assuming ergodicity, an isolated system in equilibrium can be described by a microcanonical distribution where any time-averages can be in principle calculated from the uniform probability distribution on a constant energy surface. Since, in this case, two configurations with the same position coordinates and opposite velocities, i.e, $\left\{\theta_{i}, \phi_{i}, v_{\theta_{i}}, v_{\phi_{i}}\right\}$ and $\left\{\theta_{i}, \phi_{i},-v_{\theta_{i}},-v_{\phi_{i}}\right\}$, are equally probable, the ensemble (microcanonical) average of the total magnetic moment $\sum_{i}(q / 2 c)\left\langle\left(\vec{r}_{i} \times \vec{v}_{i}\right)\right\rangle$ is identically zero.

Motion on an arbitrary curved surface - We now consider dynamics of a particle of mass $m$ and charge $q$ which is constrained to move on a closed surface of an arbitrary shape in the presence of an externally applied constant uniform magnetic field $\vec{B}=B \hat{z}$. The position of the particle in a three-dimensional space is specified by a set of general orthogonal curvilinear coordinates $\left\{q^{\mu}\right\}$ where $\mu=1,2,3$ and the respective unit vector is denoted as $\hat{q}_{\mu}$. The constraint that the particle moves on a surface can be imposed, without loss of any generality, by taking $q^{1}=$ constant or equivalently $\dot{q}^{1}=0$. Then a spatial point on the surface $q^{1}=$ constant is specified by the remaining two generalized coordinates, i.e., $\left\{q^{2}, q^{3}\right\}$. An infinitesimal line element $d l$ in the curvilinear coordinate can be written as

$$
(d l)^{2}=\sum_{\mu, \nu=1}^{3} g_{\mu \nu} d q^{\mu} d q^{\nu},
$$

where $g_{\mu \nu}$ is the metric tensor of the curved surface, represented as a $3 \times 3$ matrix. Since the coordinate system is orthogonal, the metric tensor is diagonal, i.e., $g_{\mu \nu}=\left(h_{\mu}\right)^{2} \delta_{\mu \nu}$ where $h_{\mu}$ is called the scale factor associated with the curvilinear coordinate $q^{\mu}$ and $\delta_{\mu \nu}$ is the Kronecker-delta function. For example, in spherical coordinate considered previously, $q^{1}=r, q^{2}=\theta$ and $q^{3}=\phi$ and the respective scale factors are $h_{r}=1, h_{\theta}=r$ and $h_{\phi}=r \sin \theta$. We also define a matrix element $g^{\mu \nu}$ which is inverse of the $3 \times 3$ matrix representing the metric tensor $g_{\mu \nu}$, i.e., $\sum_{\delta=1}^{3} g^{\mu \delta} g_{\delta \nu}=\delta_{\mu \nu}$. Since the metric tensor is diagonal, the inverse is also diagonal, i.e., $g^{\mu \nu}=\left(h_{\mu}\right)^{-2} \delta_{\mu \nu}$. An infinitesimal volume element $d V$ and surface element $d A$ of the surface $q^{1}=$ constant can be written as $d V=h_{1} h_{2} h_{3} d q^{1} d q^{2} d q^{3}$ and $d A=h_{2} h_{3} d q^{2} d q^{3}$.

First we consider the deterministic motion of a single particle. Newton's equation of motion can be written in the covariant form [14,

$$
\ddot{q}^{\lambda}+\sum_{\mu, \nu=1}^{3} \Gamma_{\mu \nu}^{\lambda} \dot{q}^{\mu} \dot{q}^{\nu}=\frac{F^{\lambda}}{h_{\lambda}}
$$

where $F^{\lambda}$ is the component of an external force along unit vector $\hat{q}^{\lambda}$ and the affine connection $\Gamma_{\mu \nu}^{\lambda}$ in an orthogonal curvilinear coordinate with $g_{\mu \nu}=\delta_{\mu \nu} g_{\mu \mu}$ can be written as

$$
\Gamma_{\mu \nu}^{\lambda}=\frac{1}{2} g^{\lambda \lambda}\left[\delta_{\nu \lambda} \frac{\partial g_{\lambda \lambda}}{\partial q^{\mu}}+\delta_{\mu \lambda} \frac{\partial g_{\lambda \lambda}}{\partial q^{\nu}}-\delta_{\nu \mu} \frac{\partial g_{\mu \mu}}{\partial q^{\lambda}}\right] .
$$

Note that we do not follow the Einstein summation convention here. Now defining the velocity $v^{\lambda}$ along an unit vector $\hat{q}^{\lambda}$ as $v^{\lambda}=h_{\lambda} \dot{q}^{\lambda}$ and using the relations $\ddot{q}^{\lambda}=\left(\dot{v}^{\lambda}-\dot{h}_{\lambda} \dot{q}^{\lambda}\right) / h_{\lambda}, \dot{h}_{\lambda}=\sum_{\mu}\left(\partial h_{\lambda} / \partial q^{\mu}\right) \dot{q}^{\mu}$ in Eq. (29), we get a set of two first-order differential equations,

$$
\begin{array}{r}
\dot{q}^{\lambda}=\frac{v^{\lambda}}{h_{\lambda}} \equiv \mathcal{V}_{q^{\lambda}}, \\
\dot{v}^{\lambda}=\sum_{\mu=2}^{3} \frac{\partial h_{\lambda}}{\partial q^{\mu}} \frac{v^{\mu}}{h_{\mu}} \frac{v^{\lambda}}{h_{\lambda}}-h_{\lambda} \sum_{\mu, \nu=2}^{3} \Gamma_{\mu \nu}^{\lambda} \frac{v^{\mu}}{h_{\mu}} \frac{v^{\nu}}{h_{\nu}} \\
+\frac{1}{m} F_{\text {mag }}^{\lambda} \equiv \mathcal{V}_{v^{\lambda}},
\end{array}
$$

where $F_{\text {mag }}^{\lambda}$ is the component of the Lorentz force along the unit vector $\hat{q}^{\lambda}$ acting on the particle in the presence of a magnetic field. Note that the indices $\lambda, \mu$ and $\nu$ are specified to take values 2 and 3 only as the motion along unit vector $\hat{q}^{1}$ is not possible, i.e., $v^{1}=0$. The Lorentz force is given by $\vec{F}_{\text {mag }}=(q / c)(\vec{v} \times \vec{B})$ where $\vec{v} \times \vec{B}=\left(v^{2} \hat{q}^{2}+v^{3} \hat{q}^{3}\right) \times B \hat{z}$. Now taking the components of the Lorentz force tangential to the surface, we get $F_{\text {mag }}^{2}=(q B / c) v^{3} \cos \Theta$ and $F_{\text {mag }}^{3}=-(q B / c) v^{2} \cos \Theta$ where $\cos \Theta=\left(\hat{q}^{1} \cdot \hat{z}\right), \Theta$ being the angle between unit vectors $\hat{q}^{1}$ and $\hat{z}$. The angle $\Theta$ is a function of generalized coordinates $\left\{q^{\mu}\right\}$, i.e., $\Theta=\Theta\left(\left\{q^{\mu}\right\}\right)$. For example in spherical coordinate, using explicit expressions of the scale factor $h_{\lambda}$ and the affine connection $\Gamma_{\mu \nu}^{\lambda}$, one could check that Newton's equations of motion in Eq. (31) and (32) reproduce Eqs. (44)-(7) where in this case $\Theta=\theta$. Since the external magnetic force and the constraint-forces do not perform work on the particle, using Eq. (32), one can check that

$$
\sum_{\lambda=2}^{3} \dot{v}^{\lambda} v^{\lambda}=0=\sum_{\lambda=2}^{3} \mathcal{V}_{v^{\lambda}} v^{\lambda}
$$

implying the total kinetic energy energy $\mathcal{E}=$ $(m / 2) \sum_{\lambda=2}^{3}\left(v^{\lambda}\right)^{2}=$ const in this case. 
Alternatively, to derive Newton's equations of motion of a particle constrained to move on a curved surface, one can as well start with the Lagrangian $L=K\left(\left\{q^{\lambda}, \dot{q}^{\lambda}\right\}\right)-$ $U\left(\left\{q^{\lambda}\right\}\right)$ where $K=(m / 2) \sum_{\lambda}\left(v^{\lambda}\right)^{2}$ is the total kinetic energy and $U$ is the total potential energy where the equation of motion is given by $d\left(\partial L / \partial \dot{q}^{\lambda}\right) / d t-\partial L / \partial q^{\lambda}=0$ 15. Using the affine connection in Eq. (30), one can check that Newton's equation in Eq. (29) is indeed same as the equation derived from the Lagrangian.

The time evolution of the phase space density $\rho\left(\left\{q^{\lambda}, v^{\lambda}\right\}\right)$ in 4-dimensional phase space $\left\{q^{2}, q^{3}, v^{2}, v^{3}\right\}$ is governed by Liouville's equation,

$$
\frac{\partial \rho}{\partial t}+\sum_{\lambda=2}^{3}\left[\frac{\partial\left(\rho \mathcal{V}_{q^{\lambda}}\right)}{\partial q^{\lambda}}+\frac{\partial\left(\rho \mathcal{V}_{v^{\lambda}}\right)}{\partial v^{\lambda}}\right]=0 .
$$

A straightforward calculation shows that the timeindependent solution of Eq. (34) is given by

$$
\rho_{\mathrm{st}}\left(q^{2}, q^{3}, v^{2}, v^{3}\right)=\rho_{\text {mic }} h_{2} h_{3},
$$

where the microcanonical probability distribution $\rho_{\text {mic }}=$ $1 / \Omega(E)=$ const and the phase space volume $\Omega(E)$ of a constant energy surface is given by $\Omega(E)=\left(\int d \Lambda\right) \delta(\mathcal{E}-E)$ with $d \Lambda=\prod_{\lambda=2}^{3} h_{\lambda} d q^{\lambda} d v^{\lambda}$ the infinitesimal phase space volume-measure and $\mathcal{E}=(\mathrm{m} / 2)\left[\left(v^{2}\right)^{2}+\left(v^{3}\right)^{2}\right]$ the total kinetic energy. Liouville's equation can be cast in a manifestly covariant form in terms of a scalar density variable $\tilde{\rho}=\rho / \sqrt{g}$ where $g$ is determinant of the metric tensor [16. Then Liouville's theorem of incompressibility of the phase space can be shown to have a form $d \tilde{\rho} / d t=0$. Note that Liouville's theorem holds even if one adds a positiondependent nonconservative force on the right hand side of Eq. (32), since $\partial \dot{v}^{\lambda} / \partial v^{\lambda}=0$ in this case. This will be required later to prove the nonequilibrium fluctuation theorems.

The Langevin equation in the presence of additional external conservative forces, derivable from a scalar potential $U\left(\left\{q^{\lambda}\right\}\right)$, can be written as

$$
\begin{array}{r}
\dot{q}^{\lambda}=\mathcal{V}_{q^{\lambda}}, \\
\dot{v}^{\lambda}=\mathcal{V}_{v^{\lambda}}-(\vec{\nabla} U)_{q^{\lambda}} / m-\gamma v^{\lambda}+\sqrt{2 \gamma k_{B} T / m} f_{q^{\lambda}},
\end{array}
$$

where $(\vec{\nabla} U)_{q^{\lambda}}=\left(1 / h_{\lambda}\right)\left(\partial U / \partial q^{\lambda}\right)$ is the component of the conservative forces along the unit vector $\hat{q}^{\lambda}$ and $f_{q^{\lambda}}$ is a white-noise satisfying $\left\langle f_{q^{\lambda}}(t) f_{q^{\lambda^{\prime}}}\left(t^{\prime}\right)\right\rangle=\delta_{\lambda \lambda^{\prime}} \delta\left(t-t^{\prime}\right)$. The Fokker-Planck equation, as in the case of a single particle on a sphere in Eq. (19), can be written as

$$
\begin{array}{r}
\frac{\partial P}{\partial t}=-\sum_{\lambda=2}^{3}\left[\frac{\partial\left(\mathcal{V}_{q^{\lambda}} P\right)}{\partial q^{\lambda}}+\frac{\partial\left(\mathcal{V}_{v^{\lambda}} P\right)}{\partial v^{\lambda}}\right] \\
+\sum_{\lambda=2}^{3} \frac{\partial\left(\frac{1}{m}(\vec{\nabla} U)_{q^{\lambda}} P\right)}{\partial v^{\lambda}}+\sum_{\lambda=2}^{3} \frac{\partial}{\partial v^{\lambda}}\left[\gamma v^{\lambda} P+\frac{\gamma k_{B} T}{m} \frac{\partial P}{\partial v^{\lambda}}\right] .
\end{array}
$$

Proceeding as before, the time-independent solution of Eq. (38) is given by the equilibrium ansatz $P_{\text {st }}=\rho_{\text {st }} \rho_{\text {can }}$ with $\rho_{\mathrm{st}}=\left(h_{2} h_{3}\right)$ the time-independent solution of Eq. (34) and the canonical probability distribution $\rho_{\mathrm{can}}\left(\left\{q^{\lambda}, v^{\lambda}\right\}\right)$,

$$
\rho_{\text {can }}\left(\left\{q^{\lambda}, v^{\lambda}\right\}\right)=\left(e^{-\mathcal{E} / k_{B} T}\right) / Z
$$

where $\mathcal{E}=\left[(m / 2) \sum_{\lambda}\left(v^{\lambda}\right)^{2}+U\left(\left\{q^{\lambda}\right\}\right)\right]$ and the partition function $Z=\prod_{\lambda}\left(\int h_{\lambda} d q^{\lambda} d v^{\lambda}\right) \exp \left(-\mathcal{E} / k_{B} T\right)$. The average magnetic moment can be shown to be zero since $\left\langle v^{\lambda}\right\rangle=0$ with this canonical distribution. Note that here we have considered under-damped Langevin dynamics on a curved surface. For over-damped Langevin dynamics on a curved surface and the corresponding covariant formulation of the Fokker-Planck equation, see [17,18].

In the many-particle case in a microcanonical set up, one can generalize Eq. (27) to a system consisting of particles moving on a surface of an arbitrary shape and obtain the microcanonical distribution,

$$
\rho_{\text {mic }}\left(\left\{q_{i}^{\lambda}, v_{i}^{\lambda}\right\}\right)=1 / \Omega(E)=\text { constant } .
$$

The total phase space volume $\Omega(E)$ of the constant energy surface in $4 N$-dimensional space is given by $\Omega(E)=$ $\prod_{i, \lambda}\left[\int\left(h_{\lambda} \mathrm{d} q_{i}^{\lambda}\right) \mathrm{d} v_{i}^{\lambda}\right] \delta(\mathcal{E}-E)$, where $i$ is the particle index, $\mathcal{E}=\left[(m / 2) \sum_{i, \lambda}\left(v_{i}^{\lambda}\right)^{2}+U\left(\left\{q_{i}^{\lambda}, v_{i}^{\lambda}\right\}\right)\right]$ is the total energy of the many-particle system. Since the total energy $\mathcal{E}$ is an even function of velocity $v^{\lambda}$, two configurations, one with $\left\{q_{i}^{\lambda}, v_{i}^{\lambda}\right\}$ and other with $\left\{q_{i}^{\lambda},-v_{i}^{\lambda}\right\}$, are equally probable, and this implies that the average total magnetic moment is zero for the microcanonical distribution. Adding dissipation and a noise term satisfying the fluctuation-dissipation theorem to the deterministic Newton's equation of motion of the $i$-th particle, one obtains the Langevin equation which again can be shown leading to the canonical distribution in the long time limit.

The Jarzynski equality and the Crooks theorem - We finally discuss two remarkable relations called the Jarzynski equality (JE) [6] and the Crooks theorem (CT) [7], which involve fluctuations of work done on a system driven arbitrarily far away from equilibrium.

First we consider the Langevin dynamics of a particle, in the presence of a time-dependent external magnetic field and nonconservative forces $\vec{F}_{n c}$ which include a nonconservative electric field $-\partial \vec{A} / \partial t$ induced by the time-varying magnetic field $\vec{B}=(\nabla \times \vec{A}), \vec{A}$ being the vector potential. The Langevin equation (36) still holds and, now adding the component of $\vec{F}_{n c}$ along unit vector $\hat{q}^{\lambda}$ to Eq. (37), we get

$$
\dot{v}^{\lambda}=\mathcal{V}_{v^{\lambda}}-\frac{1}{m}(\vec{\nabla} U)_{q^{\lambda}}+\frac{1}{m} F_{n c}^{\lambda}-\gamma v^{\lambda}+\sqrt{\frac{2 \gamma k_{B} T}{m}} f_{q^{\lambda}} .
$$

Let us consider a process in a symmetric time interval $-\mathcal{T}<t<\mathcal{T}$ where $\vec{F}_{n c}$ is nonzero only in a subinterval $0 \leq t \leq \tau$ with $\tau \ll \mathcal{T}$ so that, at $t= \pm \mathcal{T}$, the system is described by the canonical distribution as in Eq. (39). The work $W$ done by the external forces and the heat $Q$ transferred from the heat bath to the system can be 
written as

$$
\begin{array}{r}
W=\int_{-\mathcal{T}}^{\mathcal{T}}\left(\sum_{\lambda} F_{n c}^{\lambda} v^{\lambda}+\frac{\partial U}{\partial \alpha} \dot{\alpha}\right) d t \\
Q=\int_{-\mathcal{T}}^{\mathcal{T}} m\left(-\gamma v^{\lambda}+\sqrt{2 \gamma k_{B} T / m} f_{q^{\lambda}}\right) v^{\lambda} d t
\end{array}
$$

where $\alpha(t)$ is an external time-dependent parameter in the potential energy $U$ which varies only in the interval $0 \leq t \leq \tau$. Under simultaneous reversal of time and the direction of the magnetic field, the total work done by the external forces is odd, i.e., $W \rightarrow-W$ as $t \rightarrow-t, v^{\lambda} \rightarrow-v^{\lambda}$ and $\vec{B} \rightarrow-\vec{B}$ (equivalently $\vec{A} \rightarrow$ $-\vec{A})$ since $F_{n c}^{\lambda}$, which includes $-\partial \vec{A} / \partial t$, does not change sign. The ratio of the probability $\mathcal{P}_{F}$ of a forward path $\left\{q^{\lambda}(t), v^{\lambda}(t) ; \alpha(t), F_{n c}^{\lambda}(t)\right\}$ to the probability $\mathcal{P}_{R}$ of a reverse path $\left\{q^{\lambda}(-t),-v^{\lambda}(-t) ; \alpha(-t), F_{n c}^{\lambda}(-t)\right\}$ is given by

$$
\frac{\mathcal{P}_{F}}{\mathcal{P}_{R}}=\exp \left[-\beta \int_{-\mathcal{T}}^{\mathcal{T}} \sum_{\lambda} \mathcal{F}^{\lambda} v^{\lambda} d t\right]=\exp (-\beta Q)
$$

where $\mathcal{F}^{\lambda}=\left[m \dot{v}^{\lambda}+(\vec{\nabla} U)_{q^{\lambda}}-F_{n c}^{\lambda}\right], \beta=1 / k_{B} T$ and we have used Eqs. (33), (41) and (43). With the total energy of the system $\mathcal{E}=\left[(m / 2) \sum_{\lambda}\left(v^{\lambda}\right)^{2}+U\right]$, and by using Eqs. (33) and (41)-(43), we get $\Delta \mathcal{E}=\int(d \mathcal{E} / d t) d t=W+Q$ where $\Delta \mathcal{E}$ the change in the total energy of the system. The probability distribution $P_{F}(W)$ of work $W_{F}$ done for the forward protocol $\left\{\alpha(t), \vec{F}_{n c}(t)\right\}$ can be related to the probability distribution $P_{R}(W)$ of work $W_{R}$ done for the reverse protocol $\left\{\alpha(-t), \vec{F}_{n c}(-t)\right\}$ with the direction of $\vec{B}$ also reversed,

$$
\begin{array}{r}
P_{F}(W)=\sum_{\text {forward paths }} \rho_{i} \mathcal{P}_{F} \delta\left(W_{F}-W\right) \\
=\sum_{\text {reverse paths }} \rho_{f} \mathcal{P}_{R} \delta\left(W_{R}+W\right) \times\left(e^{-\beta Q} \frac{\rho_{i}}{\rho_{f}}\right) \\
=e^{\beta(W-\Delta F)} P_{R}(W),
\end{array}
$$

where the subscripts $i, f$ denote the corresponding initial and final quantity respectively. The canonical probability distribution of the system $\rho=\exp [-\beta(\mathcal{E}-F)]$ is given in Eq. (39) with $F=-(1 / \beta) \ln Z$ being the free energy, $\Delta F=F_{f}-F_{i}$, and we have also used Eq. (44), $\mathcal{E}_{f}-\mathcal{E}_{i}=$ $\Delta \mathcal{E}=W_{F}+Q$ and $W_{F}=-W_{R}$. The relation in Eq. (45) is the statement of the CT which has been shown here to be valid even for a particle on a curved surface. The JE, $\langle\exp (-\beta W)\rangle=\exp (-\beta \Delta F)$, can be obtained by integrating the $\mathrm{CT}$ [7. It is straightforward to extend this analysis to a many-particle system governed by Langevin dynamics on a curved surface.

In a microcanonical set up, the JE and the CT have been recently proved for a system consisting of particles moving in Euclidean space in the presence of a time-dependent magnetic field and other nonconservative forces. For particles moving on a curved surface, the proof essentially follows from the fact that Liouville's theorem still hold for the system and the heat bath combined in the presence of a magnetic field and other nonconservative forces [12].

Summary - We have shown that the classical Langevin dynamics for charged particles on a closed curved surface in a time-independent magnetic field leads to the canonical distribution in the long time limit. Thus the Bohr-van Leeuwen theorem holds even for a finite system without any boundary and the average magnetic moment for such classical systems is zero. Our analytical results disproves the recent claim by Kumar and Kumar [2] that a classical charged particle on the surface of a sphere governed by Langevin dynamics in the presence of a magnetic field has a nonzero average diamagnetic moment. We also show that nonequilibrium fluctuation theorems hold for a system consisting of particles on a curved surface in the presence of a time-dependent magnetic field and other nonconservative forces.

$$
* * *
$$

The authors thank K. Vijay Kumar for discussions and correspondence. Financial support from the DFG for project SE $1119 / 3$ is gratefully acknowledged.

\section{REFERENCES}

[1] Van Vleck J. H., The Theory of Electric and Magnetic Susceptibilities (Oxford University Press, London) 1932.

[2] Kumar N. and Kumar K. V., EPL, 86 (2009) 17001.

[3] Jayannavar A. M. and Kumar N., J. Phys. A: Math. Gen., 14 (1981) 1399.

[4] Dattagupta S. and Singh J., Phys. Rev. Lett., 79 (1997) 961.

[5] Kaplan T. A. and Mahanti S. D., EPL, 87 (2009) 17002.

[6] Jarzynski C., Phys. Rev. Lett., 78 (1997) 2690.

[7] Crooks G. E., Phys. Rev. E, 60 (1999) 2721.

[8] C. Bustamante, J. Liphardt and F. Ritort, Phys. Today, 58 (2005) 43.

[9] R. J. HARris and G. M. Schütz, J. Stat. Mech.: Theor. and Exp., P07020 (2007).

[10] Seifert U., Eur. Phys. J. B, 64 (2008) 423.

[11] Saha A. and Jayannavar A. M., Phys. Rev. E, 77 (2008) 022105.

[12] Pradhan P., Phys. Rev. E, 81 (2010) 021122.

[13] Risken H., The Fokker-Planck Equation (SpringerVerlag, Berlin) 1984.

[14] Weinberg S., Gravitation and Cosmology: Principles and Applications of The General Theory of Relativity (John Wiley and Sons, New York) 1971.

[15] Casey J., Am. J. Phys., 62 (1994) 836.

[16] Tuckerman M. E., Mundy C. J., and Martyna G. J., EPL, 45 (1999) 149.

[17] Graham R., Z. Phys. B, 26 (1977) 397.

[18] Christensen M., J. Comput. Phys., 201 (2004) 421. 